\title{
Determination of a U-Notch Stress Concentration Factor Using Thermoelasticity
}

\author{
R. B. Vieira ${ }^{1}$, S. Kurunthottikkal Philip ${ }^{2}$, G. L. G. Gonzáles ${ }^{1}$, José L. F. Freire ${ }^{1}$, B. Yang ${ }^{3}$ and R. E. Rowlands $^{2}$ \\ 1. Department of Mechanical Engineering, Pontifical Catholic University of Rio de Janeiro, PUC-Rio, Rio de Janeiro 22453-900, \\ Brazil
}

2. U. Wisconsin-Madison, USA

3. BIT, China

\begin{abstract}
This paper uses the TSA (thermoelastic stress analysis) technique to determine the stress concentration factor (Kt) of a U-notch in an aluminum plate, and then compares the results with those obtained from a FEA (finite elements analysis) of the same specimen. In order to do so, it devises a calculation procedure to extrapolate the thermoelastic data near the tip of the notch and then applies the resulting algorithm to seven distinct experiments that had different loading frequencies, mean loads and load ranges. The overall positive results suggest that the technique may be suitable for Kt measurements in real-world structures. A discussion about the calibration factor of the thermoelastic data is included by confronting the calibration results using independent tensile uniaxial tests and using the U-notch TSA and FEA paired specimen data.
\end{abstract}

Key words: Thermoelasticity, infrared, TSA, stress concentration, U-notch.

\section{Introduction ${ }^{1}$}

Thermoelastic Stress Analysis (TSA or Thermoelasticity) is an experimental stress analysis technique based on the thermoelastic effect, which describes the stress-induced change in temperature that occurs at a point on a body when submitted to elastic deformation under adiabatic conditions. Weber described the thermoelastic effect for the first time in 1830 and Lord Kelvin published the relevant theory in 1853 [[1]].

The thermoelastic effect causes a point on a body that is under cyclic loading to undergo a change in temperature that is proportional to the change in the first stress invariant, or the sum of the principal stresses. Under adiabatic and plane stress conditions, Eq. (1) holds, and describes the expected change in temperature:

$$
\Delta T=-\alpha T_{o}\left(\Delta \sigma_{1}+\Delta \sigma_{2}\right) \frac{1}{\rho c_{p}}
$$

Corresponding author: José L. F. Freire, associate professor, Ph.D., research fields: structural integrity and experimental stress analysis. where, $\Delta T$ is the change in temperature, $\alpha$ is the linear thermal expansion coefficient, $T_{\mathrm{o}}$ is a reference temperature, $\Delta \sigma_{1}$ and $\Delta \sigma_{2}$ are the ranges of the principal cyclic stresses, $\rho$ is the density, and $c_{p}$ is the specific heat coefficient at constant pressure [2]. It should be noted that the adiabatic condition will only exist for a point where the heat conduction rate is negligible in relation to the variation in temperature induced by the thermoelastic effect.

Eq. (1) is often rewritten using a material property $\mathrm{K}$, which results in Eq. (2).

$$
\Delta T=-K T_{o}\left(\Delta \sigma_{1}+\Delta \sigma_{2}\right)
$$

where, $K=\alpha / \rho . c_{\sigma}$.

Typical commercial systems designed to conduct TSA tests use infrared cameras to measure miniscule changes in temperature along the lines of about 0.001 ${ }^{\circ} \mathrm{C}$. In order to achieve such minute resolutions, the surface of the body is usually coated with a thin, uniform layer of paint having a high emissivity to behave like a black body [2].

Calibration of the thermoelastic data output may be accomplished by either bonding strain gages to 
uniform and well known regions of the specimen's stress field, or by conducting a separate calibration test using a controlled specimen made of the same material, with the same coating and under known stress field conditions; for example, a cyclic uniaxial load.

After calibration, the typical equation used in TSA is obtained [3].

$$
\Delta \sigma=A \times S
$$

where, $\Delta \sigma$ is the range of the cyclic first stress invariant under proportional loading, $\mathrm{S}$ is the raw signal from the TSA system, and A is the calibration coefficient that includes the material properties, the reference temperature and the detector parameters.

Fig. 1 shows a typical TSA system, consisting of a servo-hydraulic testing machine that applies the cyclic loading to the specimen, the load cell that feeds the loading data to the software for data acquisition, the infrared camera used to record the temperature variation signal, and the computer that correlates the signals in order to apply Eq. (3).

The ability of TSA to obtain whole stress field information makes it a highly suitable method for analysing high gradient cases such as near notch-tip or even near crack-tip stress fields. Other relevant advantages of the method are the ease of application on actual real-world components and the small amount of preparation required for the specimen. Disadvantages are the relatively high cost of the commercial systems, the necessity of a cyclic loading condition, the need to separate principal stresses from its invariant, and the experimental calibration process [2].

Recent publications regarding TSA have focused on applications, including detecting and studying stress fields around crack tips, studying stress fields in non-isotropic materials, measuring residual stresses and combining TSA with other experimental techniques such as photoelasticity and DIC (digital image correlation) [3-6].

The study of stress concentration factors $(\mathrm{Kt})$ is highly important because the high stress concentration geometric features are usually the critical points of real-world structures where fatigue cracks tend to initiate and ultimately cause a component to fail. Rowlands et al. have published a series of papers concerning the determination of full stress fields near geometric discontinuities. Several of these papers employ an Airy stress function to separate the individual stresses from the measured invariant and to determine the stresses on the edges of the components [7-11].

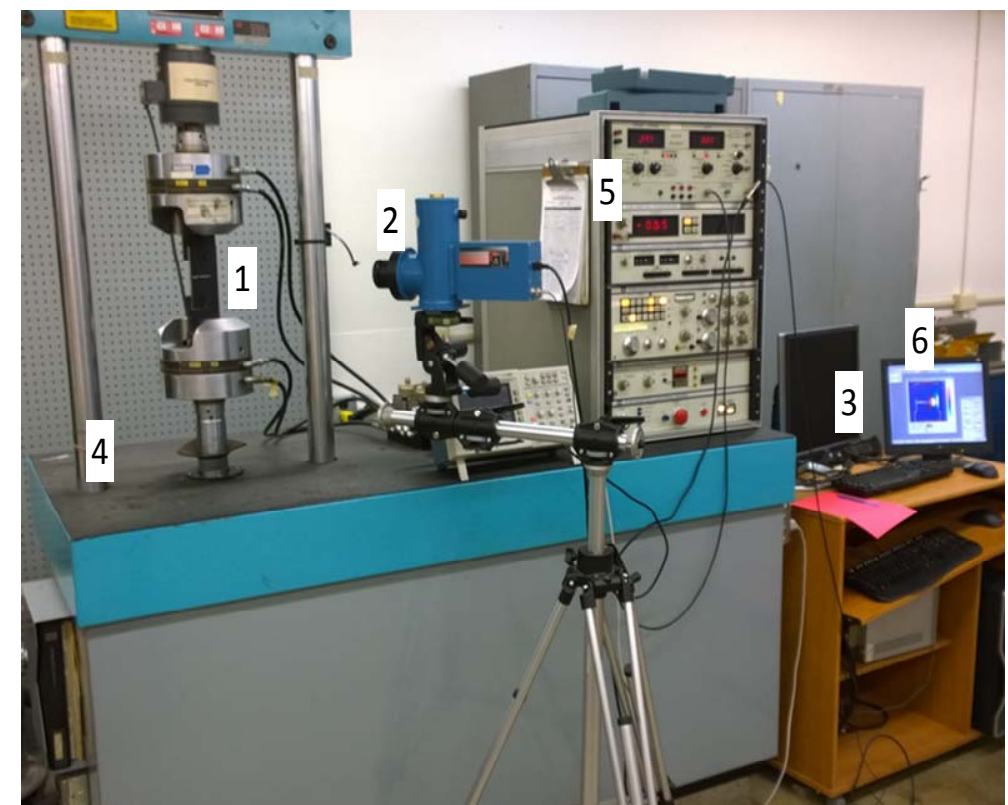

Fig. 1 Typical TSA system: (1) specimen; (2) infra-red camera; (3) computer to control lock-in and analyse image data; (4) load-frame; (5) servo-hydraulic testing machine control panel; and (6) TSA image of loaded specimen. 
The present paper analyses the possibility of using TSA to determine the stress concentration factor in a U-notched aluminium plate using a simple function for data fitting and extrapolating boundary stress values. A laboratory experiment was conducted using seven distinct mean load, frequency and load range combinations. The TSA results are compared with those from a simple finite element analysis. The experimental procedure, the post-processing and the extrapolation algorithm necessary for evaluating $\mathrm{Kt}$ are described in the next section.

\section{Experimental Procedure}

Seven experiments were conducted on a U-notched aluminum plate under uniaxial loading in order to determine the $\mathrm{Kt}$ caused by geometric discontinuity. The need to use different combinations of mean load, loading frequency and load range is due to reported potential dependences of the thermoelastic "constant" (the calibration coefficient A of equation (3)) on these factors $[12,13]$. Table 1 lists the seven different test conditions.

The column "Zoom" in Table 1 indicates whether the infrared camera was fully zoomed in on the notch tip or not, and the "Background" column indicates if a black paper background was used behind the specimen in order to facilitate the process of finding the specimen's borders.

Fig. 2 shows the dimensions of the U-notched aluminium plate and the coated specimen (Krylon Ultra-Flat Non Reflective black paint) already mounted on the MTS test machine.

As seen in Fig. 1, the system consisted of an MTS servo-hydraulic test machine, a Stress Photonics

Table 1 List of the seven test conditions.

\begin{tabular}{llllll}
\hline $\mathrm{n}$ & Frequency $(\mathrm{Hz})$ & Mean load $(\mathrm{N})$ & Load range $(\mathrm{N})$ & Zoom & Background \\
\hline 1 & 5 & 356 & 356 & no & no \\
2 & 30 & 534 & 178 & no & no \\
3 & 20 & 1,602 & 534 & yes & yes \\
4 & 20 & 1,602 & 534 & yes & no \\
5 & 30 & 534 & 178 & yes & nes \\
6 & 30 & 1,068 & 356 & yes & no \\
7 & 30 & 1,602 & 534 & & no \\
\hline
\end{tabular}

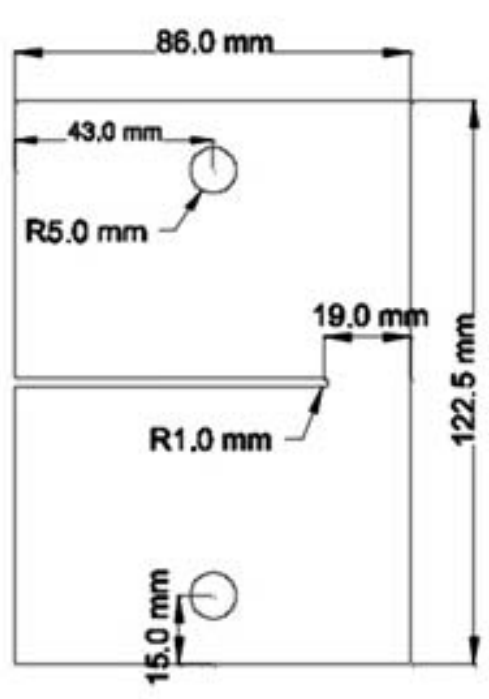

a

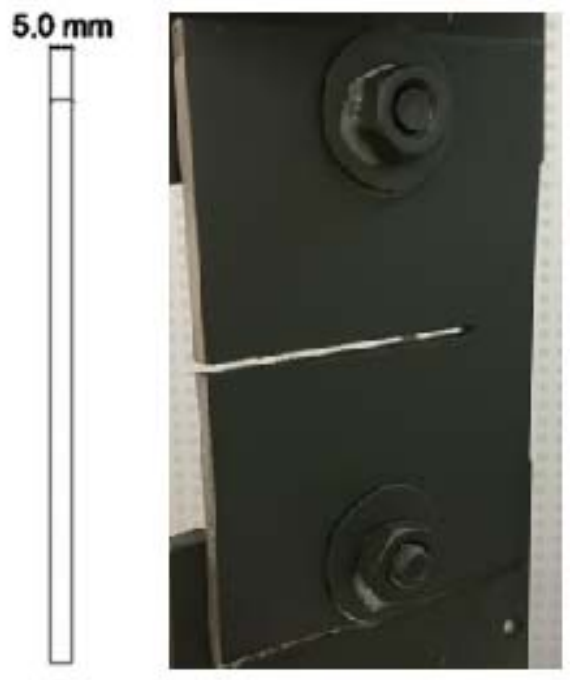

b

Fig. 2 (a) Specimen dimensions and (b) Coated specimen mounted on test machine. 
DeltaTherm DT1410 camera with a resolution of 256 $\times 256$ pixels, and a computer running the Dela Vision Analysis Software (DVAS).

A calibration experiment was conducted prior to the actual tests, using a simple uniaxially loaded aluminium plate with the same coating as the one used in the tests, having the camera in the same position, and maintaining the same temperature conditions. Next, the calibration coefficient from Eq. (3) was determined to be 0.0047 . This value is used to convert the thermoelastic data into stress invariant data from here on. Later, in Section 5 of this paper, it is presented a discussion regarding this uniaxial calibration approach and other possible calibration methods that can be used to determine the value of the calibration coefficient. These other method compare the TSA data acquired from the experiments with finite element analysis results obtained for the tested U-notch specimen.

\section{TSA Kt Results, Post-processing and Analysis}

All procedures described from here on are applied to all the tests, but all the plots shown are for the test number "4" in Table 1.

\subsection{Raw TSA Results and Specimen Border Discussion}

From recorded temperature information, the DVAS was used to provide maps of the "R" data and "Phase" data, as shown in Fig. 3 below. These maps can be translated into $256 \times 256$ matrices giving a value for each pixel of the image. The interpretation of this data is as follows: " $R$ " is the absolute value of the actual temperature signal from the system and the "Phase" value contains the information of the phase angle between the loading signal and the temperature signal. In other words, the "Phase" data is what makes it possible to determine the signal of the stress invariant, which means whether or not a point is under tensile or compressive stress in a uniaxially loaded component.
The thermoelastic data can be seen as a complex variable $\mathrm{R}=\mathrm{X}+\mathrm{iY}$, where $\mathrm{R}^{2}=\mathrm{X}^{2}+\mathrm{Y}^{2}, \mathrm{X}=\mathrm{R} \cdot \cos ($ Phase $)$ and $\mathrm{Y}=\mathrm{R} \cdot \sin ($ Phase). The maps for the $\mathrm{X}$ and $\mathrm{Y}$ values are also obtainable from the DVAS and may be combined with one another to determine the signal of the stress invariant. The absolute values of the system response " $\mathrm{R}$ " and the raw data " $\mathrm{S}$ " introduced as defined in Eq. (3) are identical, and from now on they will be represented by the data variable "S" corrected by the "Phase" signal.

The procedure used here to find the edges of the specimen and the notch tip starts by finding line AA as shown in Fig. 3. In order to do so, the highest value of " $\mathrm{S}$ " in the $256 \times 256$ matrix is found, and the line on which it belongs is the critical line of the specimen. The plot of "R", already corrected by the "Phase" signal, i.e. "S", for the full central line is shown in Fig. $4 a$.

The next step is to identify what is actually part of the specimen surface. The points between the two peaks (positive and negative) are clearly part of the plate. They are, respectively, very close to the notch tip and to the opposite border, where the maximum uniaxial tensile and compressive stresses occur. In order to consider the boundary effect, it is necessary to determine what points are still on the surface of the aluminium plate but have lower signals than the peaks. The procedure is easily understood from Fig. $4 \mathrm{~b}$.

The notch tip was defined as the boundary between the first two pixels with "R" or "S" signals above the background level, counting left from the peak pixel. The argument for this is that all the pixels between the peak and the tip (pixels 1 to 6 in Fig. 4b) are affected by the boundary, while only one pixel of air (pixel-1 in Fig. 4b) is affected since heat transfer is much more efficient in aluminum than in air. Barone and Patterson reported data with similar boundary effects in Refs. [14, 15].

A similar approach was carried out to find the opposite boundary of the specimen, and the plot of only the points that are actually on the specimen 


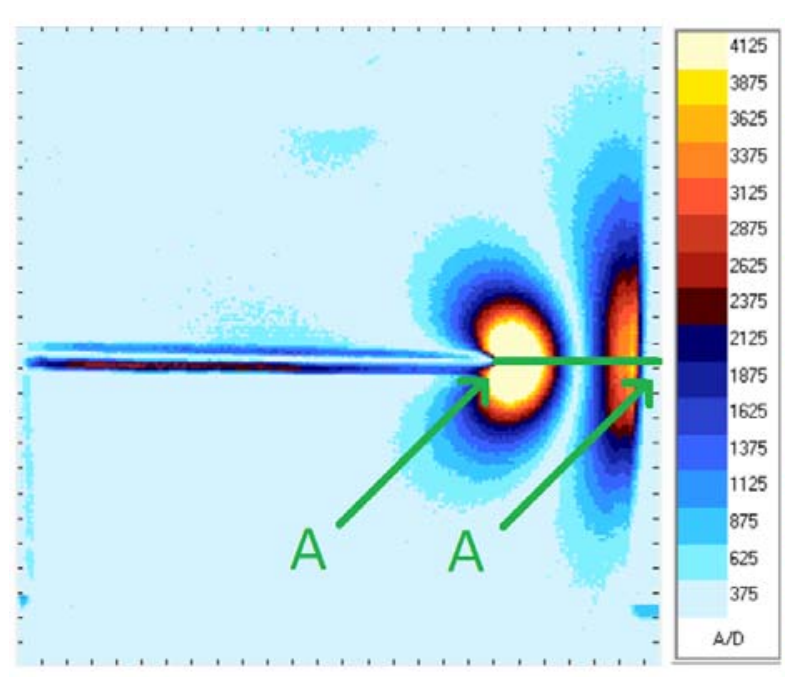

a

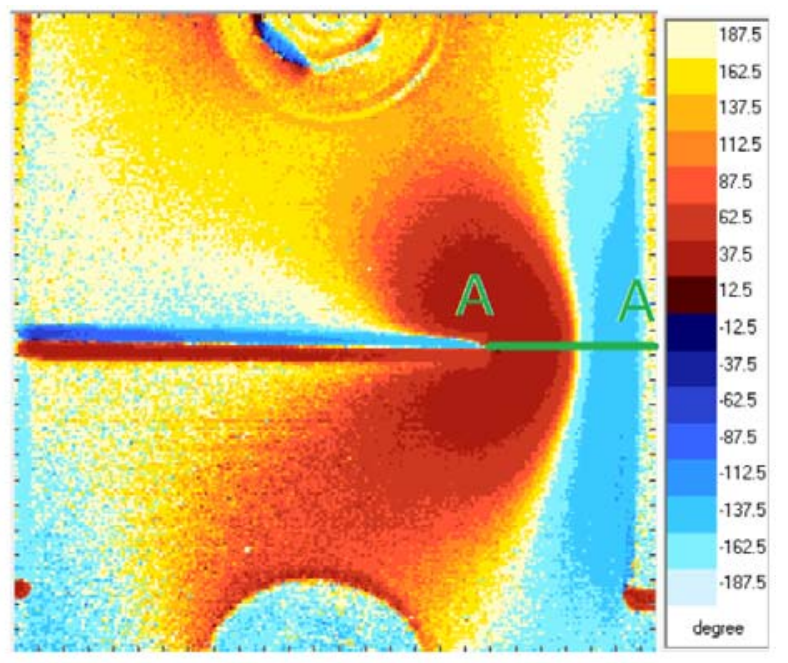

b

Fig. 3 (a) ' $R$ ' map result (b) "Phase" map result.

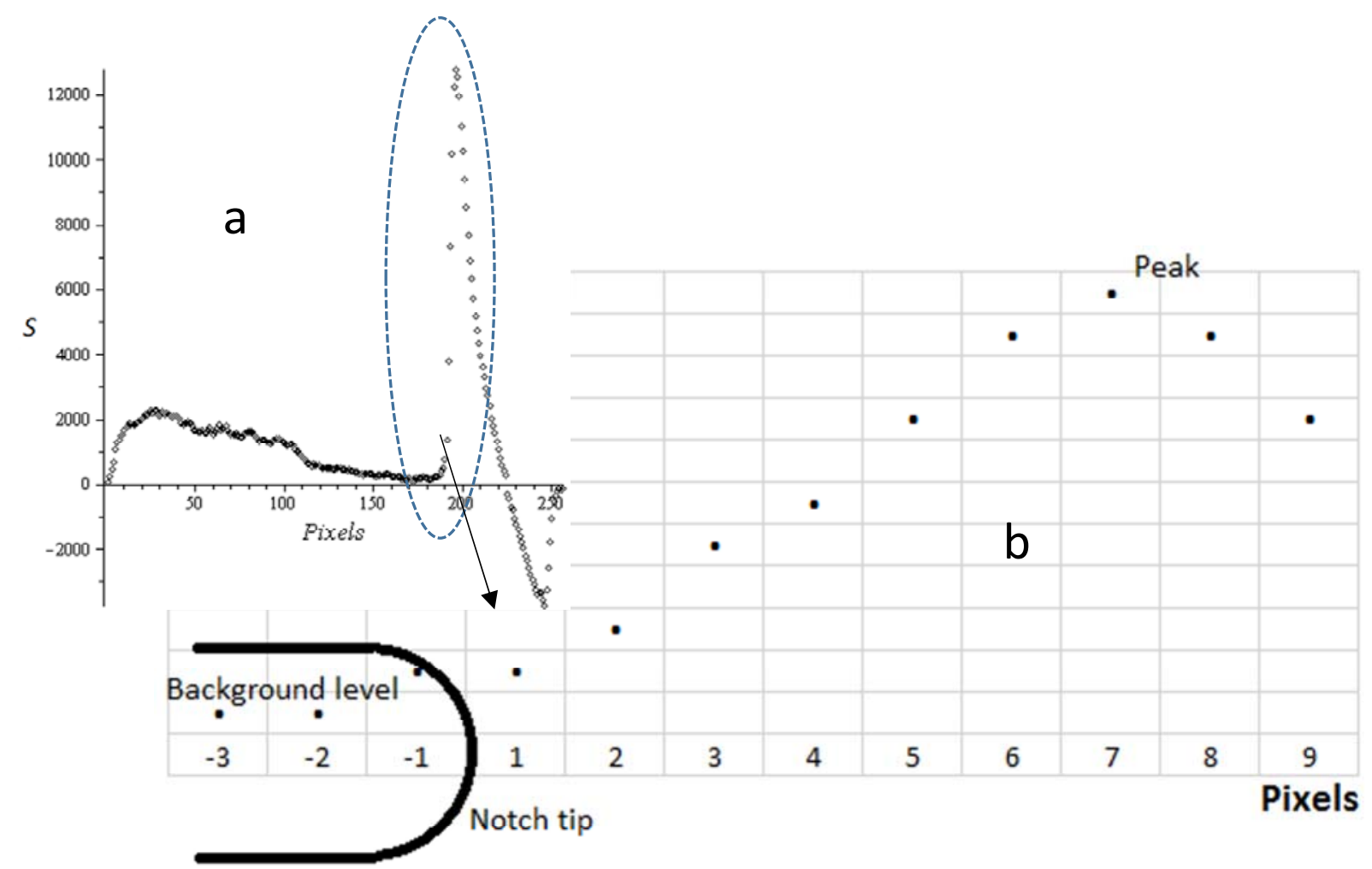

Fig. 4 (a) Raw thermoelastic signal "S" along the direction defined by the critical line AA of the specimen. Units are camera or system units (Cam units) for "S" and pixels for data point position "P"; (b) location of the notch tip, pixel counting being reset to start at the notch boundary.

surface along line AA is shown in Fig. 5, in this case with the vertical and horizontal axes units already expressed in terms of stress (MPa) and length (mm) units, respectively. For this, the axes of the plot in Fig. $4 \mathrm{a}$ are transformed by using the pixel size and the calibration coefficient found previously (0.0047). The pixel size is determined by dividing the number of pixels on the horizontal axis by the dimension of $19 \mathrm{~mm}$, which is the net section of the specimen. The result is plotted in Fig. 5. 


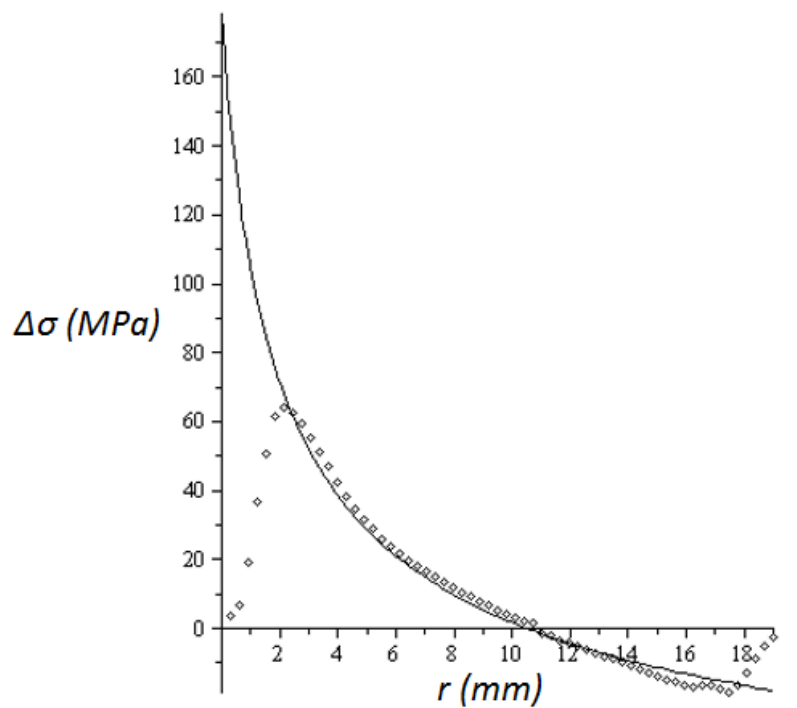

Fig. 5 Invariant stress range $\Delta \sigma(\mathrm{MPa})$ along distance $r$ $(\mathrm{mm})$ from notch tip and fitting and extrapolation of the thermoelastic data.

\subsection{Fitting and Extrapolation of the Data}

In order to calculate the stress concentration factor, it is necessary to extrapolate the data and find the stress near the notch tip. To do so, the equation used to fit the data was:

$$
\Delta \sigma=C+\frac{D}{(a+r)^{0.5}}
$$

where $\mathrm{C}$ and $\mathrm{D}$ are the fitting coefficients and $\mathrm{r}$ (in $\mathrm{mm}$ ) is the distance from the notch tip. The " $\mathrm{a}=1 \mathrm{~mm}$ " that is added to $r$ is due to the radius of the notch.

The choice of Eq. (4) is based on the fact that the U-notch is deep; therefore, the stress distribution around and close to its tip is similar to the stress field around a fatigue crack tip. The solution of Westergaard [16] for the stress field around a crack tip predicts the $1 / \mathrm{r}^{0.5}$ term, as does the Creager and Paris [17] development for estimating stress concentration factors from stress intensity factors. The result of the extrapolation is plotted in Fig. 5.

\subsection{Nominal Stress and Kt Evaluation}

In order to determine the $\mathrm{Kt}$, all one has to do is calculate the nominal stress and then use Eq. (5):

$$
K t=\frac{\sigma_{\max }}{\sigma_{\text {nom }}}
$$

Table 2 Kt results for each TSA test condition.

\begin{tabular}{ll}
\hline $\mathrm{N}$ & $\mathrm{Kt}$ \\
\hline 1 & 3.09 \\
2 & 2.84 \\
3 & 3.13 \\
4 & 2.74 \\
5 & 2.66 \\
6 & 2.34 \\
7 & 2.90 \\
Mean & 2.81 \\
Std & 0.29 \\
\hline
\end{tabular}

The nominal stress is defined as the stress under which the point of maximum stress would be if there were no stress concentration effect. Then, $\sigma_{\text {nom }}$ is the sum of the normal $\left(\sigma_{\mathrm{N}}\right)$ and bending $\left(\sigma_{\mathrm{B}}\right)$ stresses at that point, calculated as indicated in Eqs. (6) and (7), respectively.

$$
\begin{gathered}
\sigma_{N}=\frac{\Delta P}{A}=\frac{534}{19 * 5}=5.6 M P a \\
\sigma_{M}=\frac{M y}{I}=\frac{534 *(43-9.5) * 9.5}{5 * 19^{3} / 12}=59.5 \mathrm{MPa}
\end{gathered}
$$

Using Eq. (5) and the $\sigma_{\max }$ of $178 \mathrm{MPa}$ from Fig. 5:

$$
K t=2.74
$$

The results for each of the tests presented in Table 1 are shown in Table 2.

\section{Finite Elements Analysis for Kt Determination}

For comparative purposes, a 2D FEA (finite elements analysis) simulation was performed using the ANSYS commercial program, in order to evaluate the results found in the previous section. The meshed specimen is illustrated in Figs. $6 \mathrm{a}$ and $6 \mathrm{~b}$ and the results for the critical line (Line AA from Fig. 3) ahead of the notch are shown in a normalized plot in Fig. 6c.

It is now possible to get the value of $\mathrm{Kt}$ from the FEA using the same value of $\sigma_{\text {nom }}$ as before, divided by the load $P(534 \mathrm{~N})$ and the value of $\sigma_{\max }$ obtained from Fig. $6 c$ in Eq. (5).

$$
K t=\frac{0.35}{0.122}=2.85
$$




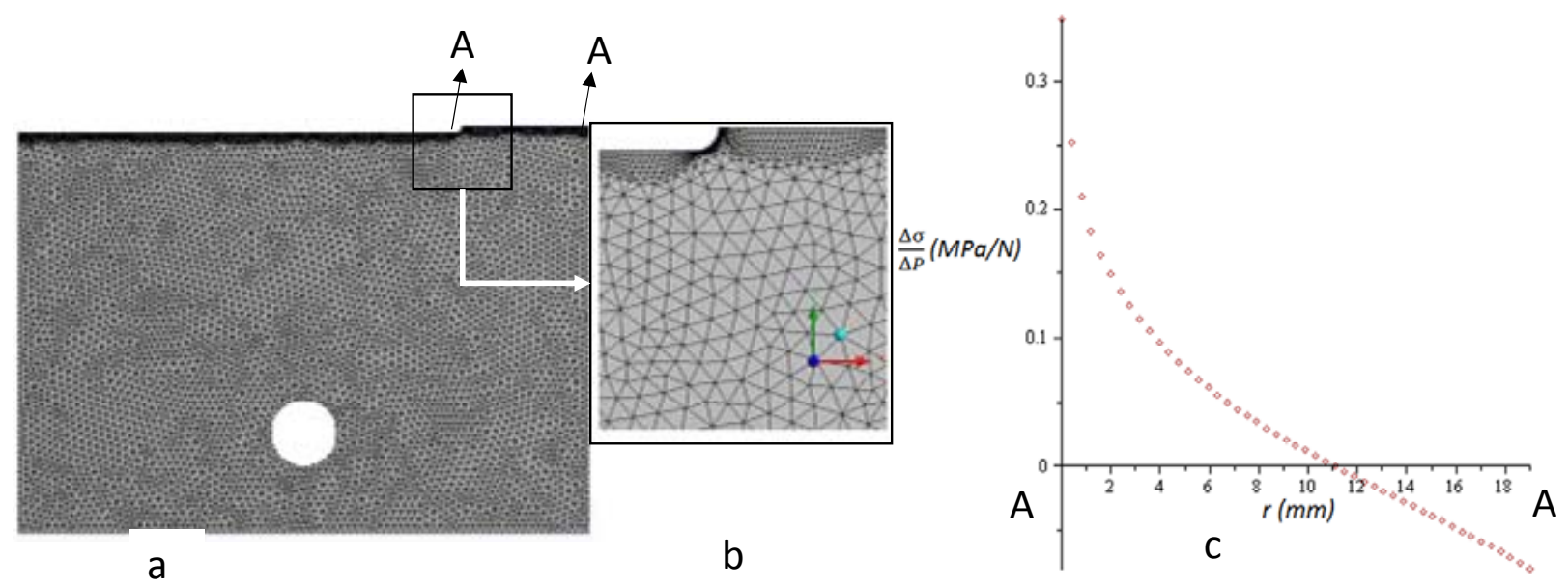

Fig. 6 (a) Meshed specimen in the FEA; (b) detail of the mesh near the U-notch; and (c) normalized plot of the FEA invariant $\Delta \sigma / \Delta \mathrm{P}(\mathrm{MPa} / \mathrm{N})$ along distance $\mathbf{r}(\mathrm{mm})$ for the critical line ahead of the notch.

The result obtained in the simulation compares well with the ones obtained with the TSA data combined with the extrapolation method used. The experimental results for Kt presented in Table 2 have a mean of 2.81, close to the FEA result, and a standard deviation of 0.29 , which is approximately equal to $10 \%$ of the mean. This is acceptable, but the somewhat high dispersion could be due to the variability of the testing conditions and analysis premises, such as: different image zooming, loading frequency and its influence on the accuracy of the temperature measurement, spurious mean stress influence on the TSA method, experimental error due to manual setting of load range and mean values, and eventual uncertainty caused by the uniaxial TSA calibration method used. It is beyond the scope of this paper to discuss in detail the influence of those parameters on the dispersion of the results. Nevertheless, the random influence of those parameters on the results of the seven experimental tests might explain the reasonable (centered) result obtained for the mean Kt value.

\section{Discussion on Calibration}

The calibration coefficient used so far in this paper was determined in the traditional way, that is, by means of tensile test repetitions applied to an aluminium bar under the same test conditions to which the U-notched specimen was submitted. The tension bar dimensions were: $260 \mathrm{~mm}$ total length, 29 $\mathrm{mm}$ grip length, $50 \mathrm{~mm}$ width and $6 \mathrm{~mm}$ thickness. The test conditions and details as well as the calibration factors determined in these tests are given in Table 3.

Although the calibration methodology described above is sufficiently robust for most experimental cases, the present investigation data offer an opportunity for discussing ways for conducting self-calibration tests. Other calibration protocols are possible if the known FE stress analysis results are coupled with the TSA data acquired for each experiment. The idea behind this discussion is to use the stress distributions obtained from the FEA of the U-notch specimen and to compare them with equivalent values obtained from the TSA experiments. There are a number of possible ways of doing these comparisons and some of them will be discussed herein.

Fig. 7 shows all the thermoelastic data obtained from the seven tests plotted in a single normalized graph. Fig. 8 shows seven curves that fitted each test acquired datum presented in Fig. 7. The fittings used Eq. (4) and the methodology presented in section 3.2 of this paper. The results shown in Figs. 7 and 8 were normalized by each test load range $\Delta \mathrm{P}$. 
Table 3 Calibration tensile tests.

\begin{tabular}{lllll}
\hline Calibration test & Frequency $(\mathrm{Hz})$ & Mean stress $(\mathrm{MPa})$ & Stress range $(\mathrm{MPa})$ & $\begin{array}{l}\text { Calibration coefficient A } \\
\left(\mathrm{MPa} / \text { cam units } \times 10^{-4}\right)\end{array}$ \\
\hline Cal 1 & 30 & 12.4 & 19.3 & 4.76 \\
$\mathrm{Cal}$ 2 & 20 & 12.4 & 19.3 & 4.72 \\
$\mathrm{Cal}$ 3 & 10 & 12.4 & 19.3 & 4.61 \\
$\mathrm{Cal} 4$ & 5 & 12.4 & 19.3 & 4.59 \\
Cal average & - & - & - & 4.67 \\
Cal dispersion & - & - & - & 0.1 \\
\hline
\end{tabular}

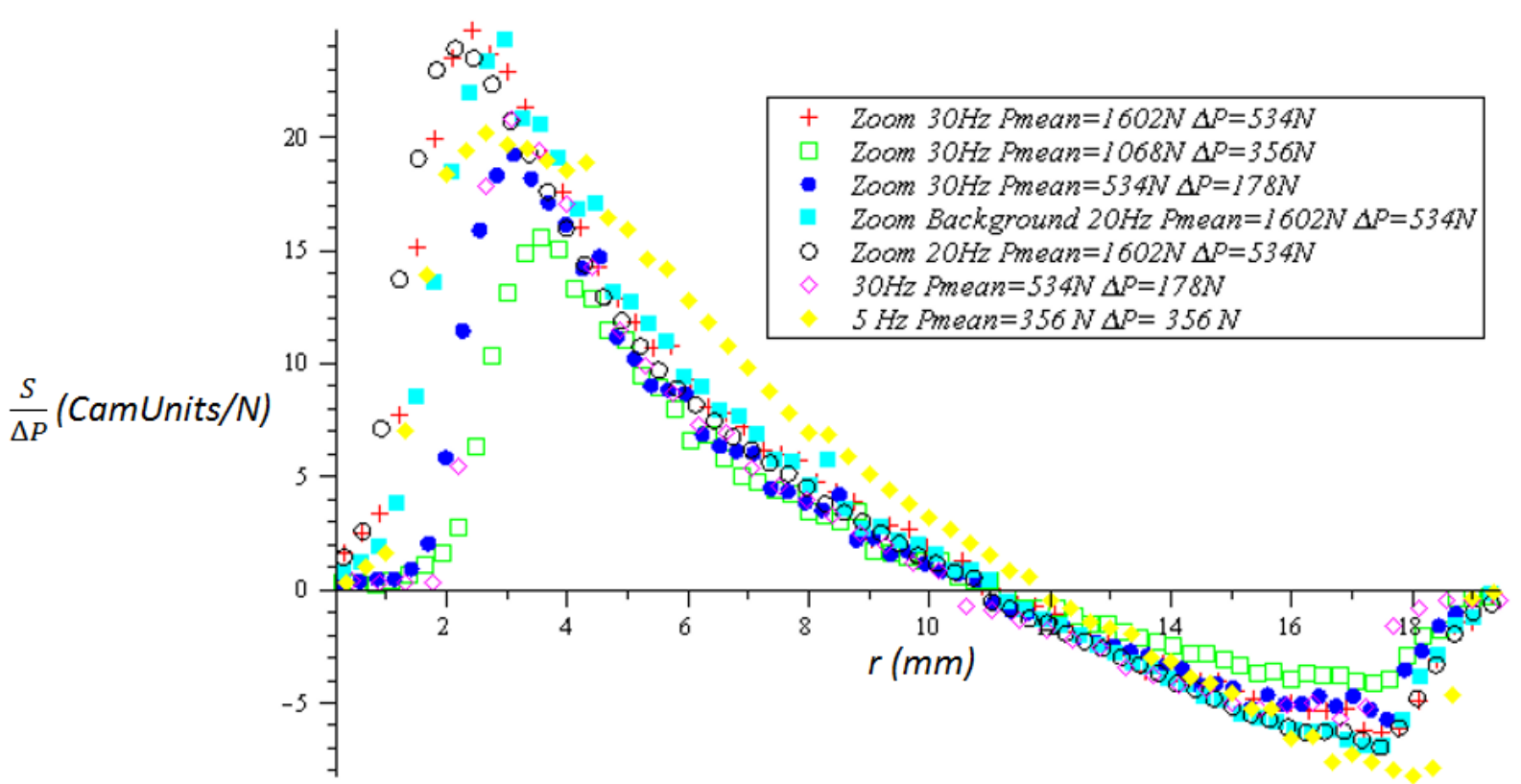

Fig. 7 Normalized TSA data $\mathrm{S} / \Delta \mathrm{P}$ (Cam units/N) for all tests plotted against position $\mathbf{r}(\mathrm{mm})$ from the notch tip along line AA (Fig. 3).

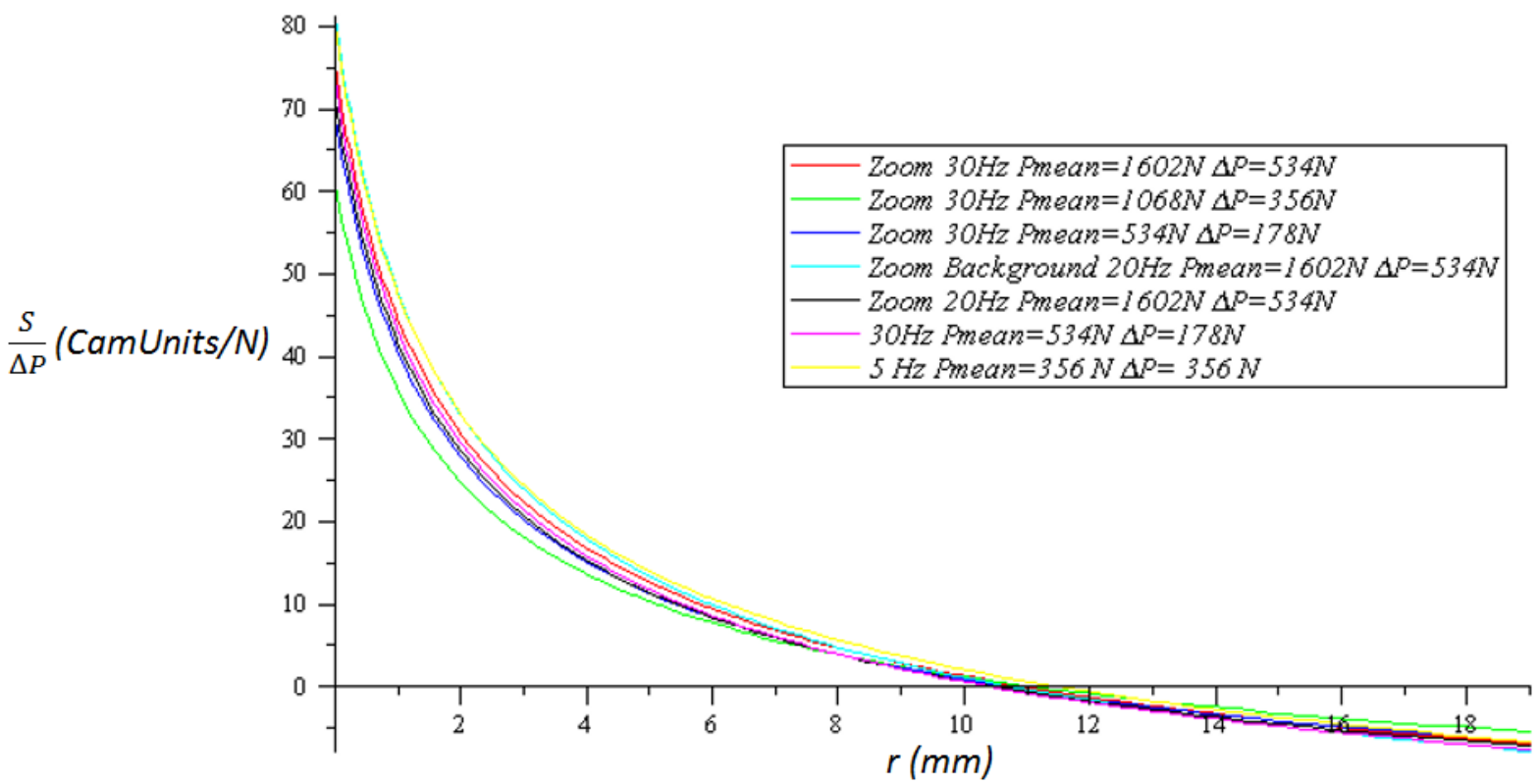

Fig. 8 Normalizedfitting curves determined for each test. Normalized TSA data S/AP (Cam units/N) for all tests plotted against position $\mathbf{r}(\mathrm{mm})$ from the notch tip along line AA. 
Table 4 Results for the calibration coefficient at the notch tip $A_{0}$.

\begin{tabular}{ll}
\hline $\mathrm{n}$ & $\mathrm{A}_{0}$ \\
\hline 1 & 0.0040 \\
2 & 0.0044 \\
3 & 0.0040 \\
4 & 0.0046 \\
5 & 0.0047 \\
6 & 0.0053 \\
7 & 0.0043 \\
Mean & 0.0045 \\
Std & 0.00045 \\
\hline
\end{tabular}

Besides the first calibration method (Table 3), a second method entails using the normalized extrapolated stress values for the notch root from each of the fitting curves, and then comparing them with the notch root stress value determined from the normalized FE solution. The values calculated at the notch tip position (" $\mathrm{A}_{0}$ ") of coefficient " $\mathrm{A}$ " of equation (3) are listed in Table 4 below. They are very similar to the value of 0.0047 previously obtained from the tensile calibration tests.

A third calibration method also makes use of the Eq. (4) fitting curve. It fits a global set of all valid TSA normalized data to a unique fitting curve, as presented in Fig. 9, and uses the extrapolated value at the notch root to determine a unique stress concentration factor $\mathrm{Kt}=2.87$ (same methodology as in Section 3.4) and to determine the calibration factor " $\mathrm{A}_{0} "=0.00456$. This factor is obtained by making equal the normalized TSA extrapolated value at the notch root and the FE value for the same point. The global set of valid TSA data used in the overall curve-fitting excludes all measured points that are affected by the specimen's boundaries. The points included in the unique curve fitting process include points located between the $4 \mathrm{~mm}$ and $17 \mathrm{~mm}$ distances from the notch root. These valid points and the fitting curve are presented in Fig. 9.

A forth way of determining a calibration factor does not use data fitting curves for the invariant stress distributions. Instead, it uses the so-called valid TSA normalized data defined in the paragraph above and their equivalent or paired point-by-point normalized finite element results along line AA. A plot of these paired points (TSA, FEA) is presented in Fig. 10.

An interesting observation from Fig. 10 is that the point-by-point paired comparison shows two different fitting slopes: one for positive (tensile stresses) and the other for negative (compressive stresses) invariant ranges. The slope coefficient for compressive

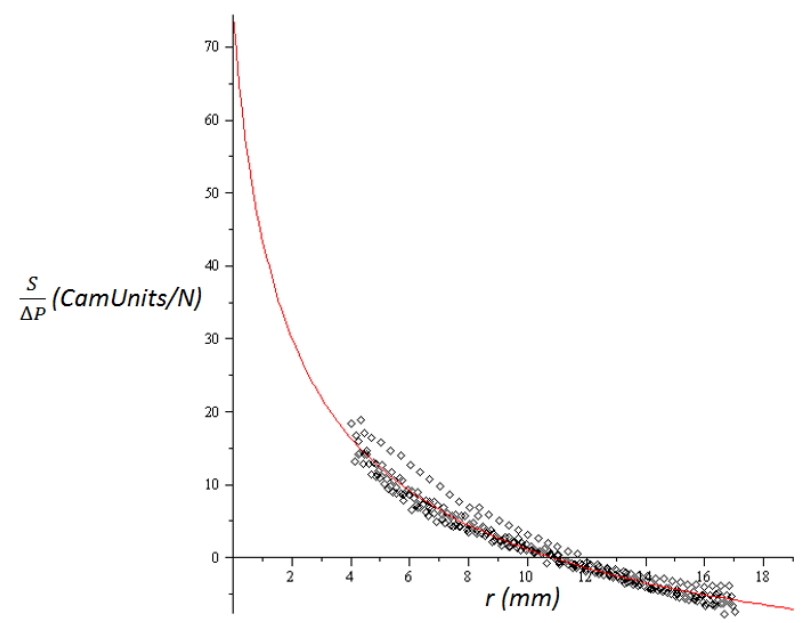

Fig. 9 Unique normalized fitting curve determined for all data points located between the $4 \mathrm{~mm}$ and $19 \mathrm{~mm}$ distances from the notch tip along line AA.

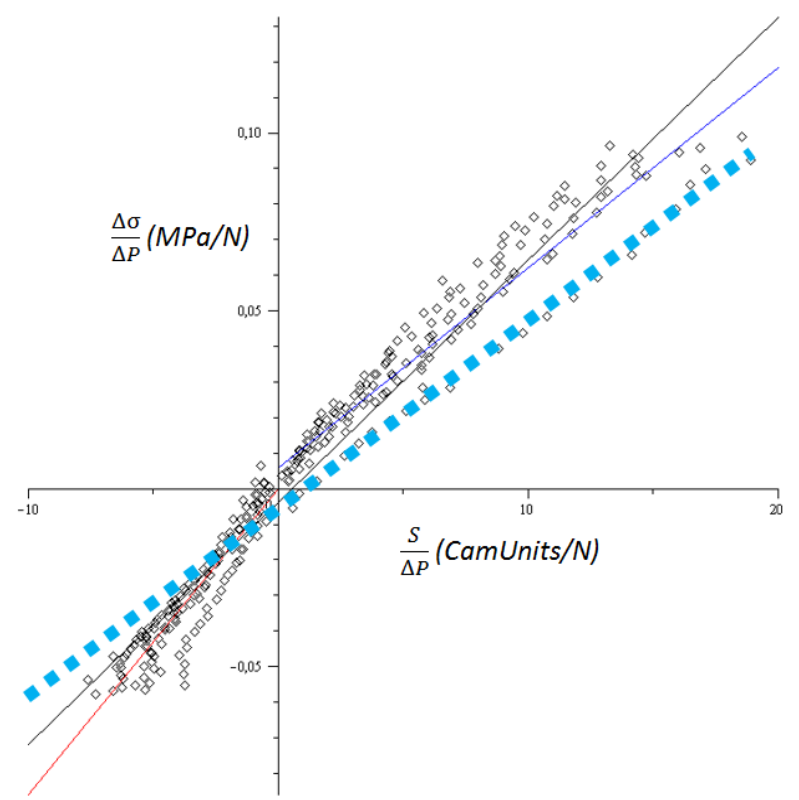

Fig. 10 Plot of FEA against TSA normalized paired data. The straight lines represent fitting of compressive, tensile, overall and average coefficient determined from the tensile tests (dotted line). Their slopes are equal to $0.0086,0.0056$, 0.0068 and 0.0047 , respectively. 
invariant stress ranges $(0.0086)$ is greater than that for tensile invariant stress ranges $(0.0056)$. The composed coefficient (all points in the plot) is equal to 0.0068 . The dotted straight line plotted in Fig. 10 represents the average calibration factor 0.0047 , determined from the tensile test calibration procedure presented at the first. Differences in slopes or calibration factors between positive and negative invariant ranges are not expected in traditional TSA theory. Therefore, the above slope differences are subject to ongoing investigation. In this, consideration is given to the influence of test variables on data measurement, such as differences caused by the magnitude of load range values used in the calibration experiments, the influence of $3 \mathrm{D}$ effects, the influence of mean stress $[12,13]$, and the camera response to high or low stress values associated with test load frequency. For the present analysis, the calibration factor of 0.0047 determined by the independent tensile calibration test could be used as the benchmark result, with which other coefficients would be compared. In this way, it can be seen that the spread of the tensile invariant data points shown in Fig. 10, resulting in the line with slope 0.0056, completely envelopes the tensile calibration coefficient straight line (0.0047 slope).

\section{Conclusions}

Based on the finite elements analysis results, the experiments carried out in this paper show that the thermoelastic stress analysis technique is suitable for evaluating stress concentration factors. The proposed simple curve fitting and extrapolation procedure produced good mean results (less than 5\%) for determining $\mathrm{Kt}$, thus proving it to be a robust method for estimating stresses near edges from TSA data.

The proposed analysis of the calibration coefficient shows no significant dependence on test conditions such as mean load, loading frequency or load range. It also demonstrates an interesting possibility that the coefficient for compression and tension stresses could be slightly different.

\section{References}

[1] Thomson, W. (Lord Kelvin). 1853. "On the Dynamical Theory of Heat."Trans. R. Soc (Edin) 20: 161-283.

[2] Dulieu-Barton, J. M. 1999. "Introduction to Thermoelastic Stress Analysis." Strain 35 (2): 35-9.

[3] Patki, A. S., and Patterson, E. A. 2010. "Thermoelastic Stress Analysis of Fatigue Cracks Subject to Overloads." Fatigue FractEngng Mater Struct 33: 809-21.

[4] Tomlinson, R. A., Ying, D., and Patterson, E. A. 2011. "Understanding Crack Tip Plasticity-A Multi-experimental Approach." Applied Mechanics and Materials 70: 153-8.

[5] Diáz, F. A., Yates, J. R., and Patterson, E. A. 2004. "Some Improvements in the Analysis of Fatigue Cracks Using Thermoelasticity." International Journal of Fatigue 26: 365-76.

[6] Robinson, A., Dulieu-Barton, J. M., Quinn, S., and Howarth, D. 2013. "Crack Detection in Large Welded Components under Fatigue Using TSA.” In Proceedings of Annual Conference on Experimental and Applied Mechanics, Society for Experimental Mechanics, SEM.

[7] Samad, W. A., and Rowlands, R. E. 2014. “On Improving Thermoelastic Stress Analysis Data near Edges of Discontinuities." In Proceedings of Annual Conference on Experimental and Applied Mechanics, Society for Experimental Mechanics, SEM.

[8] Lin, S. J., Samad, W. A., Khaja, A. A., and Rowlands, R. E. 2015. "Hybrid Thermoelastic Stress Analysis." Experimental Mechanics 55: 653-65.

[9] Samad, W. A., and Rowlands, R. E. 2014. "Full-Field Thermoelastic Stress Analysis of a Finite Structure containing an Irregularly-Shaped Hole.” Experimental Mechanics 54 (3): 457-70.

[10] Lin, S.-J., Matthys, D. R., and Rowlands, R. E. 2009. "Separating Stresses Thermoelestically in a Central Circular Perforated Plate Using an Airy Stress Function." Strain 45 (6): 516-26.

[11] Khaja, A. A., Matthys, D. R., and Rowlands, R. E. 2014. "Determing All Dispalcements, Strains and Stresses Full-Field from Measured Values of a Single Displacement Component." Experimental Mechanics 54 (3): 443-55.

[12] Machin, A. S., Sparrow, J. G., and Stimson, M. G. 1987. "Mean Stress Dependence of the Thermoelastic Constant." Strain 23 (1): 27-30.

[13] Palumbo, D., and Galietti, U. 2016. "Data Correction for Thermoelastic Stress Analysis on Titanium Components." Experimental Mechanics 56 (3): 451-62.

[14] Barone, S., and Patterson, E. A. 1998. "Polymer Coating 
as a Strain Witness in Thermoelasticty." J. Strain Analysis 33 (3): 223-32.

[15] Barone, S., and Patterson, E. A. 1996. "Full-Field Separation of Principal Stresses by Combined Thermoand Photoelasticity." Experimental Mechanics 36 (4): 318-24.
[16] Westergaard, H. M. 1939. "Bearing Pressures and Cracks." Journal of Applied Mechanics 61: 49-53.

[17] Creager, M., and Paris, P. C. 1967. "Elastic Field Equations for Blunt Cracks with Reference to Stress Corrosion Cracking." International Journal of Fracture Mechanics 3: 247-52. 